\title{
Optimization of the Tolling Level and Tolling Period for Bottleneck Road
}

\author{
REN Hualing ${ }^{1,}$, , LI Shubing ${ }^{2}$ and XUE Ying ${ }^{1}$ \\ ${ }^{1}$ MOE Key Laboratory for Urban Transportation Complex Systems Theory and Technology, Beijing \\ Jiaotong University, Beijing, 100044 \\ ${ }^{2}$ Department of Public Security, Shandong police College, Jinan, Shandong, 250014 \\ aehualingren@163.com
}

\begin{abstract}
Keywords: bottleneck model; tolling level; tolling period; Greenshields model; bi-level programming model

Abstract. This paper mainly optimizes the level and period of congestion pricing in single-step-toll method on a bottleneck link. Firstly, Greenshields model is applied to obtain the queue length and the speed of the moving part at a stable state. Then, the bi-level programming model is established, in which the upper level is to minimize the maximal queue length and the minimal speed, and the lower programming is to simulate the travel behaviors using the Greenshields model. The bi-level programming model is solved by an improved genetic algorithm, to obtain optimal pricing level and pricing period. Finally, a simple example is given to illustrate the application of the model and the algorithm.
\end{abstract}

\section{Introduction}

Nowadays, due to rapidly economic development, the rapidly growth in car ownership in most cities in the world has made them experience serious traffic congestion. Traffic congestion seriously effects people's traveling, environmental quality and the urban planning. In 1975, Singapore firstly implemented congestion pricing policy. However, due to technical limits, it was only a manual pricing. Then in 1998, Singapore became the first city that implemented electronic fee collection. In 2003, London applied the congestion pricing policy, and the pricing areas were mainly concentrated in the urban center. In 2006, Stockholm began to implement the congestion pricing policy, and after trial operation of six months, it was proved having eliminated the traffic congestion [1].

In our country, along with the rapid growth of car ownership, Beijing, Shanghai, Chengdu, and other cities have suffered different degrees of traffic congestion [2]. Means of limiting the number of travelling and buying cars by choosing number are used in Beijing to control the traffic congestion. Recently, congestion pricing methods are also discussed to be used to control the traffic congestion.

In most dynamic traffic congestion models, congestion tolls vary continuously over time to achieve an absolutely social optimum. Yet no existing road pricing scheme has such sophisticated, continuously time-varying tolls. Although the optimal time-varying toll is capable of eliminating queuing time completely, it has such practical difficulties as incomplete knowledge for toll determination and high costs for toll collection. Due to these difficulties, a step toll scheme has been considered as an alternative to reduce queuing time.

But the single-step-toll models are most analytical and are based on the assumption that the vehicles have no lengths which is unpractical and difficult to be used in pricing on the networks. This paper will establish a single-step-toll model based on the Greenshields model [3], and then optimize the tolling level and tolling period by a bi-level programming, minimizing the maximal queue length and the minimal speed. The rest of the paper is organized as follows: In Section 2, we derive a single-step-toll model based on Greenshields model. The tolling level and the tolling period are optimized using a bi-level programming model in section 3. In Section 4, a numerical example is given. Finally, our summary and conclusions are provided in Section 5. 


\section{A single-step-toll model based on Greenshields model}

In this section, a single-step-toll model based on the Greenshields model is proposed. In the model, the vehicles have normal lengths. The moving vehicles' behaviors on the road satisfy the Greenshields speed-density relationship, and the queuing vehicles occupy the road length, which is more practical to be used in pricing on the networks.

Symbols in the model. In the bottleneck model there is a continuum of $D$ identical commuters who travel alone by car (one traveler per car) from a common origin to a common destination connected by a single road which is subject to bottleneck queuing congestion. Without a queue, an individual thus departs "from home", passes the bottleneck, and completes his/her trip by arriving "at work" all at the same moment. The symbols are as follows:

$X(t)$--- the number of vehicles on the link at time $t$;

$u(t)$--- the inflow rate at time $t$;

$v$--- the outflow rate capacity of bottleneck;

$X^{2}(t)$--- the number of vehicles on the queuing section at time $t$;

$r(t)$--- the speed of moving vehicles on the link at time $t$;

$r^{\max }$--- the upper speed limit on the link;

$r^{\min }$--- the lower speed limit on the link;

$k_{j}$--- the jam density on the link;

$k^{1}(t)$--- the density of the moving vehecles on the link at time $t$;

$C(t)$--- the travel cost at time $t$;

$t(t)$--- the travel time of vehicles entering the link at time $t$;

$t^{1}(t)$--- the travel time of the moving vehicles on the link at time $t$;

$t^{2}(t)$--- the delaying time of queuing vehicles at time $t$;

$\left[\begin{array}{ll}t^{+}, & t^{-}\end{array}\right]$--- the tolling period;

$\left[w^{l}, w^{r}\right]---$ the time window of starting working;

$\alpha$--- the unit cost of travel time;

$\beta, \gamma---$ the unit cost of early and late penalty;

Greenshields model. In this paper, we only consider the bottleneck section and a certain period of time between $[0, T]$. Then the work starting time is setting as $\left[w^{l}, w^{r}\right]$. Commuters arrive at the bottleneck before $w^{l}$ will have the early penalty, and arrive at the bottleneck after $w^{r}$ will have the late penalty. To avoid serious traffic congestion, a step toll is collected during a certain period of time $\left[t^{+}, t^{-}\right]$. The dynamic link travel time is divided into two parts: moving time and queuing delay time. To calculate these two travel times, the link is also divided into two parts: moving part and queuing part. The lengths of these two parts are relevant to the time, because the queue lengths at different time are different.

When the vehicles depart on the link at time $t$, the number of vehicles on the link at this time is decided by the inflow rates at $t-1$, the capacity of bottleneck and the number of vehicles at $t-1[4$, 5]:

$$
X(t)=X(t-1)+u(t-1)-v, \forall t .
$$

Assuming the density of queuing part is equal to the jam density, then the queue length at time $t$ can be calculated:

$$
L^{2}(t)=\frac{X^{2}(t)}{k_{j}}, \forall t .
$$

According to the above equations, we can get the density of the moving part at time $t$ : 


$$
k^{1}(t)=\frac{X(t)-X^{2}(t)}{L-\frac{X^{2}(t)}{k_{j}}}, \forall t .
$$

So, using the Greenshields speed-density relationship, the speed of moving part at time $t$ can be described by the density of the moving part, that is,

$$
r(t)=r^{\text {min }}+\left(r^{\max }-r^{\min }\right)\left[1-\left(\frac{k^{1}(t)}{k_{j}}\right)^{a}\right]^{b}, \forall t .
$$

The number of queuing vehicles at time $t$ is related to the number of queuing vehicles at $t-1$, the capacity of bottleneck, the speed and density of the moving part at $t-1$ :

$$
X^{2}(t)=X^{2}(t-1)+r(t-1) \cdot k^{1}(t-1)-v, \forall t .
$$

From

$$
\begin{aligned}
& t(t)=t^{1}(t)+t^{2}(t), \forall t, \\
& t^{1}(t)=\left[L-X^{2}(t) / k_{j}\right] / r(t), \forall t,
\end{aligned}
$$

and

$$
t^{2}(t)=\left\{X^{2}(t)+\left[r(t) \cdot k^{1}(t)-v\right] \cdot t^{1}(t)\right\} / v, \forall t .
$$

we can have the travel time $t(t)$.

So the travel cost at time $t$ on the link can be obtained $[6,7,8]$ :

$$
C(t)=\left\{\begin{array}{lc}
\alpha t(t)+\beta\left[w^{l}-t-t(t)\right], & t+t(t)<w^{l} \\
\alpha t(t)+\gamma\left[t+t(t)-w^{r}\right], & t+t(t)>w^{r} \\
\alpha t(t)+0, & \text { otherwise }
\end{array}+\left\{\begin{array}{cc}
C_{t o l l}, & t \in\left[t^{+}, t^{-}\right] \\
0, & \text { otherwise }
\end{array}, \forall t .\right.\right.
$$

where $\gamma>\alpha>\beta$. $\alpha$ is the unit cost of travel time, and $\beta(\gamma)$ is the unit cost of early (late) penalty.

The commuters choose their departure time so that the following user equilibrium (UE) state is satisfied: at any chosen departure time, the commuters have the same minimal travel cost, while at any other time, the travel cost are larger than the minimal travel cost. In order to get the UE state, the inflow rates for each time are updated by the travel costs through an iterative process, which is summarized as follows:

Step 1: Set OD demand as $D$. Set tolling level $C_{\text {toll }}$ and tolling period $\left[t^{+}, t^{-}\right]$.

Step 2: Set the initial inflow rate $u(t)_{1}, \sum_{t=0}^{T} u(\mathrm{t})_{1}=D$ and the iterative number $n=1$.

Step 3: According to Eqs. (1) and (5), the number of vehicles and queuing vehicles can be obtained.

Step 4: According to Eq. (6)-(8), the travel time can be calculated.

Step 5: According to Eq (9), the travel costs at each time can be calculated. The minimal travel cost $C_{n}^{\min }$ can be found.

Step 6: The inflow rates for each time can be updated by travel costs and minimal travel cost, that is,

(1) If $C(t)_{n} \neq C_{n}^{\min }$, set

$$
u(t)_{n+1}=u(t)_{n}-\rho \cdot u(t)_{n} \cdot\left[C(t)_{n}-C_{n}^{\min }\right],
$$

(2) If $C(t)_{n}=C_{n}^{\min }$, set

$$
u(t)_{n+1}=u(t)_{n}+\frac{\sum \rho \cdot u(t)_{n} \cdot\left[C(t)_{n}-C_{n}^{\min }\right]}{\left|C^{\min }{ }_{n}\right|} .
$$

Where $\left|C_{n}^{\min }\right|$ is the number of times at which the travel cost equals the minimal travel cost. 
Step 7: If $\frac{\sum u(t)_{n} \cdot\left[C(t)_{n}-C_{n}^{\min }\right]}{\sum u(t)_{n} \cdot C^{\min }{ }_{n}}<\varepsilon$, stop; otherwise, set $n=n+1$, and return to Step 3 .

Numerical example of the Greenshields model. In this sub-section, a simple numerical example is given to show the application of the Greenshields model. The number of queuing vehicles and the speed of moving vehicles can be obtained.

Set the link length as $5.25 \mathrm{~km}$, total traffic demand as $625 \mathrm{vehicles,} \mathrm{free-flow} \mathrm{speed} \mathrm{on} \mathrm{the} \mathrm{link} \mathrm{as}$ $0.7 \mathrm{~km} / \mathrm{min}$, the minimum speed as $0.3 \mathrm{~km} / \mathrm{min}$, the travel time of free-flow speed on the link is $7.5 \mathrm{~min}$, and the crowding density $160 \mathrm{vehicles} / \mathrm{km}$, which means that the capacity of the bottleneck is 15vehicles/min (Chris et al., 2010).

In addition, other parameters include: $a=1, b=3, \alpha=1, \beta=1, \gamma=1, \rho=0.0001$, $\left[w^{l}, w^{r}\right]=[75,85],\left[t^{+}, t^{-}\right]=[55,75]$. The tolling level is set as 2 .

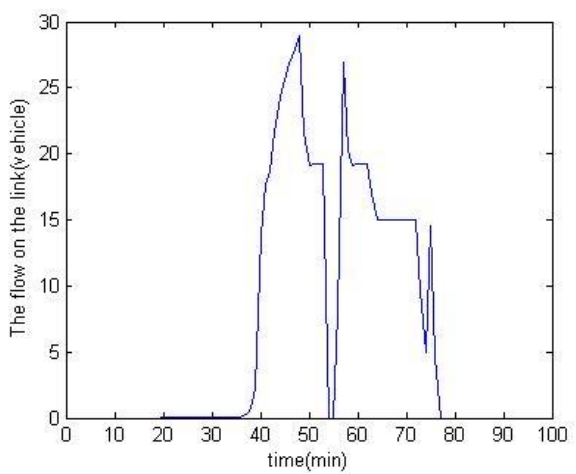

Fig.1. The inflow rate on the link

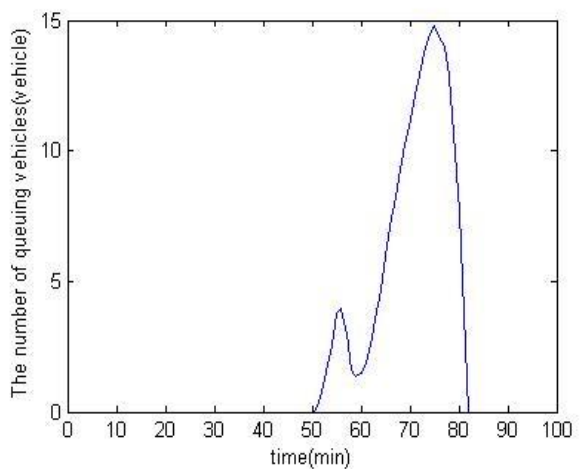

Fig.3. The number of queuing vehicles

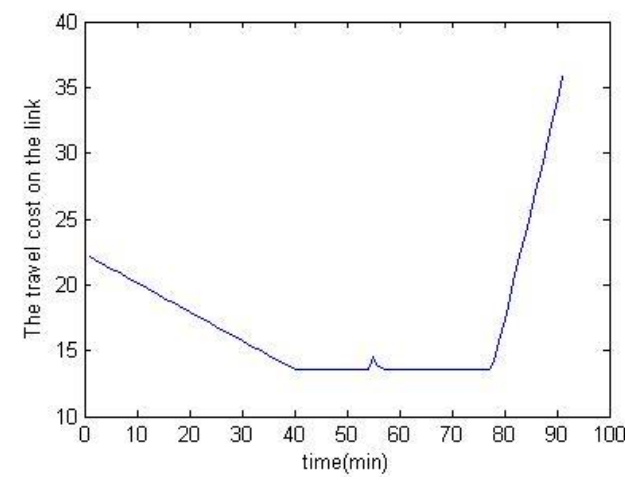

Fig.2. The travel cost on the link

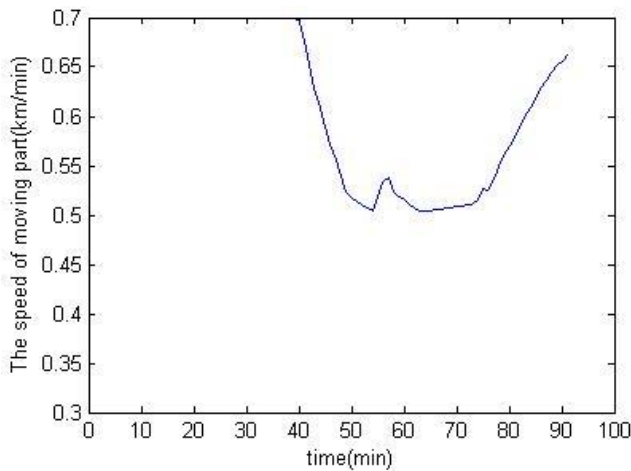

Fig.4. The speed of moving vehicles

Fig. 1-4 show the inflow rate, the travel cost, the number of queuing vehicles and the speed of moving vehicles, respectively. Because of early penalty the number of commuters departing before tolling period is relatively few. So it does not cause congestion queuing, and the speed is relatively high. When $t=t^{+}=55 \mathrm{~min}$ the tolling period begins, and the sudden toll cannot be made up by the continuous reduction in the number of queuing vehicles and the falling of the speed of moving vehicle, so there is a small time period around $t^{+}$during which the travel costs are higher than the equilibrium travel cost. During this dwell time, the higher travel cost leads to zero departing rates, and the number of queuing vehicles is decreasing and the speed of moving part is improved (see Fig. 3 and Fig. 4 during a few minutes around $t^{+}$). The inflow rates begin to return and increase rapidly after this dwell time interval. Congestion pricing stops when $t^{-}=75 \mathrm{~min}$, commuters will choose to depart after this time to reduce their travel cost. There is a sudden increasing in inflow rates, but because the queuing vehicles are already dissipated, the speed of moving vehicles is still high (as Fig.4). 


\section{The optimization model and the solution algorithm}

The optimization model. Using these relationships in the Greenshields model, the number of queuing vehicles can be minimized and the speed of moving vehicles can be maximized by optimizing the tolling level and the tolling period. So a bi-level programming is proposed in this section, and the upper objective is to both minimize the number of queuing vehicles and maximize the speed of moving vehicles. The Greenshields model is the lower level model to describe the travel behaviors under certain tolling level and tolling period.

The upper planning model is as follows:

$$
\begin{aligned}
\underset{C_{\text {toll }} t^{+} 、 t^{-}}{\min } & Z=\theta \max X^{2}(t)-(1-\theta) \mu \min r(t) \\
\text { s.t. } & C_{\text {toll }}^{\min } \leq C_{\text {toll }} \leq C_{\text {toll }}^{\max }, \\
& t^{+ \text {min }} \leq t^{+} \leq t^{+\max }, \\
& t^{-\min } \leq t^{-} \leq t^{-\max } .
\end{aligned}
$$

The values of $X^{2}(t)$ and $r(t)$ can be obtained from the Greenshields model in the lower programming. $\theta$ is the weight coefficient and $\mu$ is a conversion factor of different units between the number of queuing vehicles and the speed of moving vehicles. The different values of the weight coefficient stand for different importance of the two parts of the objective.

For any given $C_{\text {toll }} 、 t^{+}$and $t^{-}$, we can calculate $X^{2}(t)$ and $r(t)$ by the Greenshields model, which will be reflected to the upper model in the iterative algorithm, and then the upper model can decide the adjust direction of the tolling level and the tolling period.

Solution algorithm As it is known, the solution of the bi-level programming is very difficult, because it is a NP-hard problem. So some heuristic solution algorithms are adapted to solve the bi-level programming. In this paper, a genetic algorithm $[8,9]$ is presented to solve the proposed bi-level programming, which has the characteristics of high applicability, simple program, and high searching efficiency. The summary of the algorithm is as follows.

Step 1: Set the parameters in the genetic algorithm: crossover probability $p_{c}$, mutation probability $p_{m}$, maximum evolution generation MaxGen, the number of populations of each generation $m$ ( $m$ is an even number).

Step 2: Randomly generate $m$ populations $C_{\text {toll }}{ }^{(0)}, t^{+(0)}$ and $t^{-(0)}$ which satisfy the upper constraints. Set the initial evolution generation $N=0$.

Step 3: Define the upper objective function as the fitness function. Encode the upper decision variables and solve the lower Greenshields model described in subsection 2.2. Calculate the values of individual fitness. The upper decision variable of the largest fitness ranking is as the optimal solution if $N=\mathrm{MaxGen}$. Otherwise go to step 4.

Step 4: Select and copy the next generation populations $C_{\text {toll }}^{(N)}, t^{+(N)}$ and $t^{-(N)}$.

Step 5: All individuals in the populations are randomly paired, and a random number $e_{2}$ between 0 and 1 is generated. Make crossover operation if $e_{2}<p_{c}$.

Step 6: Mutation process.

Step 7: Set $N=N+1$, and return to step 3 .

\section{Numerical example}

This section provides a simple example to apply the presented model and its solution algorithm, and seeks the optimal solution based on the example given in subsection 2.3.

Firstly, the input parameters are given. Set the link length as $5.25 \mathrm{~km}$, total traffic demand as 625 vehicles, free-flow speed on the link as $0.7 \mathrm{~km} / \mathrm{min}$, the minimum speed is $0.3 \mathrm{~km} / \mathrm{min}$, and the travel time of free-flow on the link is $7.5 \mathrm{~min}$ (Chris et al., 2010). Set other parameters: crowding density is $160 \mathrm{vehicles} / \mathrm{km}$, and the capacity of the bottleneck is $15 \mathrm{vehicles} / \mathrm{min}$. In addition, working 
starting time is between $75 \mathrm{~min}$ and $85 \mathrm{~min}$ and there is no early and late arrival penalty during this period $^{[11][15]}$. According to user equilibrium theory, the lower Greenshields model reaches stable state at $n=55000$. The tolling level ranges from 1 to 10 . The starting time of tolling ranges from $40 \mathrm{~min}$ to $60 \mathrm{~min}$, and the ending time of tolling ranges from $70 \mathrm{~min}$ to $90 \mathrm{~min}$. The parameters in the genetic algorithm include $p_{c}=0.6, p_{m}=0.1, m=20$, MaxGen $=50, \theta=0.6, \mu=10$.

Given the above data, three optimized tolling schemes are considered respectively in this paper:

(1) Minimize the maximal number of queuing vehicles and maximize the minimal speed at the same time;

(2) Maximize the minimal speed of the moving vehicles only;

(3) Minimize the maximal number of queuing vehicles only.

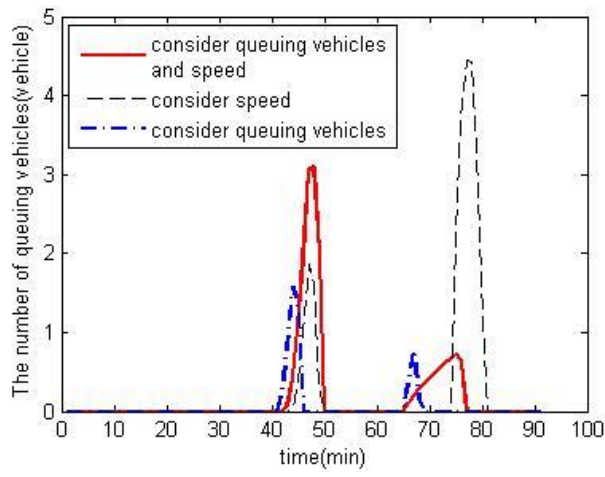

Fig.5. The number of queuing vehicles

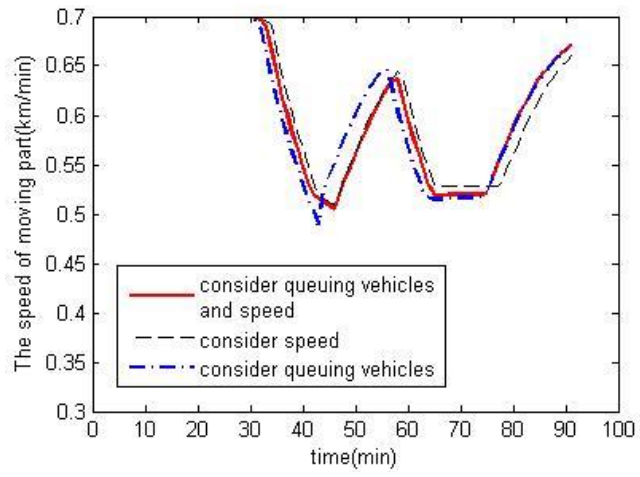

Fig.6. The speed of moving vehicles

Three optimum solutions are obtained based on the above three schemes:

(1) $C_{\text {toll }}=5, t^{+}=47, t^{-}=80$;

(2) $C_{\text {toll }}=5, t^{+}=46.46, t^{-}=73.13$;

(3) $C_{\text {toll }}=4.91, t^{+}=44, t^{-}=79$.

As shown in Fig.3 that, for scheme 2, although the minimal speed has been optimized and the minimal speed is the highest in the three schemes (Fig. 5), the maximal number of the queuing vehicles is the largest. After the end of the tolling period, there is a serious congestion. When we consider minimizing the maximal number of queuing vehicles only in the third scheme, the maximal queuing vehicles is optimized and the maximal queuing vehicles is the minimum in three schemes. However, the minimal speed of the moving part under this scheme is lower than the other two schemes. Applying the first scheme to alleviate bottleneck congestion, we can reduce the maximal number of queuing vehicles and increase the minimal speed of moving vehicles at the same time.

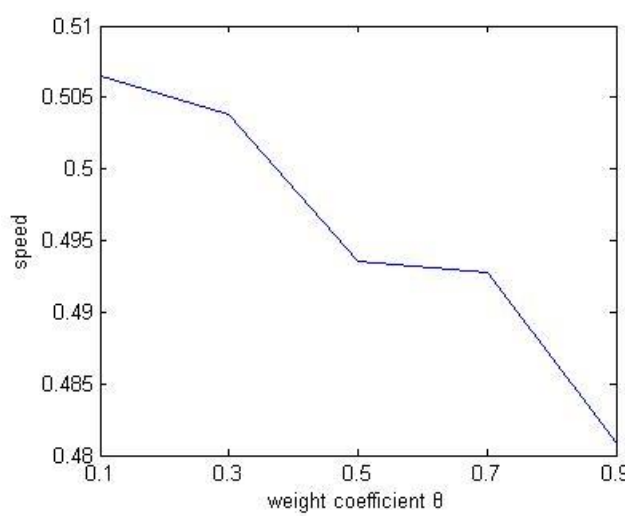

Fig.7. Minimal speed - weighting coefficient curve

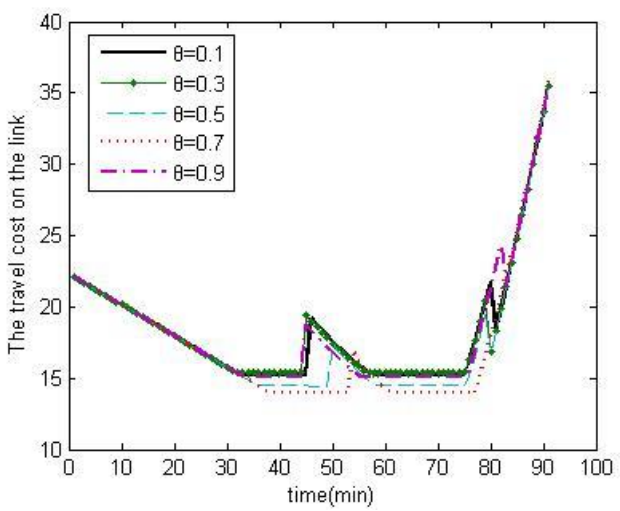

Fig.8 Travel cost - weight coefficient curve

The weight coefficient $\theta$ in the established upper model in the bi-level programming stands for the important extent of the number of queuing vehicles and the speed of moving vehicles when optimizing the tolling level and the tolling period. 
Fig.8 shows the link travel costs on the whole rush hour for different weight coefficients of $\theta$. For each $\theta$, after the last non-toll-payer departs, there is a dwell time interval during which no commuter is willing to depart, because the tolling makes a sudden rising to the travel cost.

The optimal solutions are obtained for different weight coefficients as follows:

(1) If $\theta=0.1, C_{\text {toll }}=5, t^{+}=46, t^{-}=80$;

(2) If $\theta=0.3, C_{\text {toll }}=5, t^{+}=45, t^{-}=79$;

(3) If $\theta=0.5, C_{\text {toll }}=4, t^{+}=49.16, t^{-}=79.61$;

(4) If $\theta=0.7: C_{\text {toll }}=4, t^{+}=53.47, t^{-}=73.29$;

(5) If $\theta=0.9: C_{\text {toll }}=4.35, t^{+}=44.89, t^{-}=82$.

From these optimal results, we can see different weight coefficient leads different optimal tolling levels, tolling periods and different equilibrium travel costs. Furthermore, we find that when $\theta=0.7$, the optimal tolling level is the lowest, the optimal tolling period is the shortest, and the travel cost is the smallest. That is to say, in the optimal model, it is more effective to take both the number of queuing vehicles and the speed of moving vehicles into consideration, compared to considering only one of them.

\section{Conclusions}

In this paper, a Greenshields model is applied to obtain the queue length and the speed of the moving vehicles on a bottleneck link, which is more realistic and simple to describe the travel behaviors in practice. Then a single-step-toll model is established based on the Greenshields model to show the effect of step-toll on the commuters' travel behaviors. Furthermore, a bi-level programming model is proposed, in which the upper level is to minimize the maximal number of queuing vehicles and the minimal speed of moving vehicles, and the lower programming is the single-step-toll model based on the Greenshields model. The bi-level programming model is solved by an improved genetic algorithm to obtain the optimal pricing level and pricing period. In the numerical example, three tolling schemes are optimized to illustrate the application of the model and to analyze the function of the tolling schemes.

\section{Acknowledgements}

The work was financially supported by the National Natural Science Foundation of China (71371026, 71471104, 71171124).

\section{References}

[1] Wang X W, Zhao S C. A summarization of the transportation congestion pricing in the main cities of the world [J]. Science paper online, , Vol. 3 (2008), p. 746-750.

[2] Chen N. The study on congestion charge in central urban district-Take Chengdu for an example [J]. Journal of Guangzhou University (Natural Science Edition), Vol. 8 (2009), p. 91-94.

[3] Ren H L, Gao Z Y, Lian A P. Dynamic user optimal assignment problem based on Greenshields model [C]. Traffic and Transportation Studies Proceedings of ICTTS 2006, (2006), p. 326-335.

[4] Gao Z Y, Song Y F. A reserve capacity model of optimal signal control with user-equilibrium route choice [J]. Transportation Research Part B, Vol. 36 (2002), p. 313-323.

[5] Huang H J, Lam W H K. Modeling and solving the dynamic user equilibrium route and departure time choice problem in network with queues [J]. Transportation Research Part B, Vol. 36 (2002) p. 253-273.

[6] Chris M J T, Francesco V, Lambertus H I. New Developments in Transport Planning [A]. 
Edward Elgar, (2010).

[7] Chen L R, Zhang L. Congestion Pricing Model and Algorithm Based on Bi-level Programming Model [J]. Journal of Beijing University of technology, Vol. 32 (2006), p. 526-529.

[8] Tian X M, Gong J. On overview of real-coded genetic algorithm [J]. Journal of Hunan Environment-Biological polytechnic, Vol. 11 (2005), p. 25-31.

[9] Ren H L, Gao Z Y, Lam W H K, Long J C. Assessing the benefits of integrated en-route transit information systems and time-varying transit pricing systems in a congested transit network [J]. Transportation Planning and Technology, Vol. 32 (2009), p. 215-237. 